\title{
QT Dispersion-An Ignored Screening Tool For Cardiovascular Disease In Diabetics
}

\author{
Dr. Madhugandha Wahane ${ }^{1}$,Dr. Archana Gupta ${ }^{2}$,Dr. Pranav Tyagi ${ }^{3}$,Dr. Pawan \\ Singhal ${ }^{4}$,Dr. Arif Mohammad ${ }^{5}$,Dr. Nikhil Shah ${ }^{6}$ \\ ${ }^{2}$ (Associate Professor, Department Of Medicine ,GRMC Gwalior, Madhya Pradesh, India )

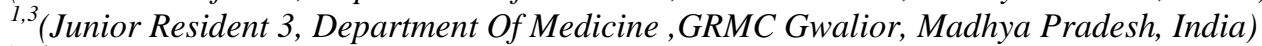 \\ ${ }^{4,5,6}$ (Junior Resident 1, Department Of Medicine ,GRMC Gwalior, Madhya Pradesh, India)
}

\begin{abstract}
Background: Diabetes Mellitus Type 2 is a major global non-communicable disease among adults causing micro and macrovascular complications, one being cardiovascular system. $Q T$ dispersion represents the summation of several adverse conditions in myocardium like fibrosis, hypertrophy, dilatation and autonomic dysfunction.

Aim and Objective: To study QT dispersion in diabetics and non-diabetics of age group 30-60 year and compare the two according to age, gender, duration of diabetes, BMI, and other clinical determinants.

Material and Methods: 100 diabetics were compared to age and sex matched 50 non diabetics. They were screened for blood sugar and QT dispersion calculated on a 12 lead ECG.

Result: QTd was significantly increased in diabetics with $p$ value -0.006. Difference of QTd between cases and controls was insignificant for different age and BMI, among gender and for dyslipidemia or presence of family history of diabetes. A positive correlation was found with longer duration of diabetes and prolonged QTd ( $p$ value-0.004).

Conclusion: QTd is an indicator for impaired myocardial electrical stability and indicates a non uniform ventricular repolarization. Present study shows presence of hyperglycemia strongly associated with prolonged QTd suggesting increased risk of arrhythmia. QTd was not affected with age, gender, BMI, dyslipidemia or presence of family history of diabetes but longer duration of hyperglycemia and poor control showed prolonged QTd. It concludes QTd as significant prognostic marker for early detection of risk of cardiovascular morbidity and can be used as screening tool to prompt further investigation.
\end{abstract}

Keyword: Type 2 diabetes mellitus, QT dispersion, cardiovascular complication, prognostic marker, screening tool.

\section{Introduction}

Type 2 Diabetes mellitus is a state of hyperglycemia giving rise to micro and macrovascular complications. With changing lifestyle there is a significant rise in diabetics all around the world further increasing the morbidity and mortality or diminished quality of life. In $1996 \mathrm{M}$. Shimaburuko et $\mathrm{al}^{1}$ first investigated association of QT dispersion with morbidity in diabetics. QTd represents summation of adverse conditions present in diabetic myocardium like fibrosis, hypertrophy, dilatation and autonomic dysfunction ${ }^{2}$. Several experimental and clinical studies show relation of QT dispersion to an increasing risk of cardiovascular morbidity and mortality. Although there is higher sensitivity and specificity of detection of cardiovascular abnormality by echocardiography and angiography but calculating QT dispersion in a simple bedside 12 lead ECG provides a quick and economical tool at hand.

\section{Material and methods}

A case control study of 100 cases and age and sex matched 50 controls in 30-60 year age group was done. All known and newly diagnosed diabetics according to ADA guideline 2015 were selected as cases. Subjects excluded were patients with atrial flutter or fibrillation, a cardiac pacemaker, AV block, Bundle branch block and frequent extrasystole, with any associated ischemic heart disease, hypertension, COPD, dyselectrolytemia, renal diseases, history of taking antihypertensive, antiarrhythmic or drugs affecting QT interval, known history of any microvascular complication like peripheral vascular disease, retinopathy and nephropathy and patients with diabetes for more than 10 year were not included. 
A detailed history of diabetes symptoms onset, treatment, risk factors of atherosclerosis or any previous atherosclerotic event was taken. History for family history of diabetes, history of smoking, alcohol was taken with thorough physical and systemic examination. A Fasting and Random blood glucose was done for all patient by a biochemical glucose peroxidase test and with glucometer. Biochemical test with standard lab technique for $\mathrm{CBC}$, lipid profile, blood urea, serum creatinine, was done. Fundus and USG abdomen was done to rule out retinopathy and nephropathy.

All data was analysed using IBM SPSS and EPI Info 7.0 software. Categorical variable expressed in percentage. Mean of statistical data was calculated and expressed as mean \pm SD. A p value of $<0.05$ was considered significant.

\section{Results}

Total 26\% of all cases and controls showed prolonged QTd. A total of 33\% diabetics and 12\% non diabetics had prolonged QTd. Mean QTd of diabetics was $45.71 \pm 2 ; 62.43 \pm 2.3$ and $63.15 \pm 2.8$ and non diabetics $43.6 \pm 2 ; 40 \pm 1.9$ and $47.6 \pm 2.2$ in different age groups of 30-40;40-50 and 50-60 respectively. This suggests that QTd was more in diabetics.

The mean age of diabetic with prolonged QTd was $50.76 \pm 6.58$ and normal QTd $46.96 \pm 9.9$ with P value 0.05 suggesting age was affecting QTd prolongation. P value for BMI was 0.91 , for positive family history was 0.47 which showed it did not significantly alter QTd. QTd prolongation with duration of diabetes had a $\mathrm{p}$ value of 0.004 which showed significant association.

\section{Discussion}

The study showed that QTd was prolonged in diabetics compared to non diabetics. It is correlating with other studies done by M Singh et al $(2001)^{3}$, Clemente et al $(2012)^{4}$, Psallas $\mathrm{M}(2006)^{5}$ amd Shimabakuro et al. ${ }^{1}$ This suggest QTd was affected by hyperglycemia, to study further QTd prolongation was seen for duration of hyperglycemia and it showed that with longer duration of hyperglycemia there was prolonged QTd. This is concurrent to studies done by Robillion (1999) ${ }^{6}$ and Neki N et al(2014) ${ }^{7}$. A study done by Veglio et al $(1998)^{8}$ also showed that poor control of diabetes mellitus as suggested with elevated $\mathrm{HbA1C}$ also caused prolongation of QTd. Takahashia et $\mathrm{al}^{9}$ and Arduino et $\mathrm{al}^{10}$, Macfarlane et $\mathrm{al}^{11}$ have previously shown that age and gender had no effect on prolonged QTd; Gupta et al(2009) ${ }^{12}$ have also shown that lipid profile did not affect QTd, this finding is similar to the finding in present study. Furthermore the present study suggested that the presence of family history of diabetes also does not prolong QTd.

\section{Conclusion}

This study looked into significance of QT dispersion among diabetics compared to nondiabetics. As QTd is a known indicator of impaired electrical stability and indicates nonuniform repolarization suggesting increased risk of arrhythmia or presence of underlying damaged myocardium. A prolonged QTd was seen with presence of hyperglycemia especially with longer duration and higher level because of poor control while age, gender, BMI, dyslipidemia or presence of family history of diabetes did not show any significant association with prolonged QTd.

This suggests that QTd should be done routinely for all patients with diabetes mellitus to rule out presence of gross cardiovascular damage. It presents as a simple, quick, cheap and resourceful but ignored screening tool especially in areas with limited resources.

\section{References}

[1]. Shimaburuko M, Chibana T, Yoshida H, Nagamine F, Komiya I, Takasu N. Increased QT dispersion and cardiac adrenergic dysinnervation in diabetic patients with autonomic neuropathy. Am J Cardiol. 1996 Nov 1; 78(9): 1057-9.

[2]. Naas A A, Davidson N C, Thompson C, Cummings F, Ogston S A, Jung R T, et al. QT and QTc dispersion are accurate predictors of cardiac death in newly diagnosed non-insulin dependent diabetes: cohort study. BMJ . 1998 Mar 7; 316 (7133): 745-6.

[3]. Singh M et al. "QT dispersion and cardiac autonomic dysfunction in patients with type 2 diabetes mellitus" JAPI 2001;49:70

[4]. Clemente D, Pereria T, Riberio S. Ventricular repolarization in Diabetic Patients: characterization and clinical implications.

[5]. Psallas M, Tentolouris N, Cokkinos A, Dimitrius P, COkkins D. QT dispersion: comparison between diabetic and non diabetic and coorelation with CAN; Hellenic J Cardiol 2006. 47; 255-262.

[6]. J.F.Robillon, J.L.Sadoul, S.Benmerabet, L.Joly-Lemoine, A.Fredenrich, B.Canivet. Assessment of cardiac arrhythmic risk in diabetic patients using QT dispersion abnormalities Diabetes \& Metabolism 1999;25:419-423.

[7]. Neki NS; Kaur J. A study of QTc prolongation and QT dispersion (QTd) as an indicator of cardiac autonomic neuropathy(CAN) in Type 2 Diabetes Mellitus patients; JIMSA Oct-Dec 2014. Vol 27 no.4 p 195-196.

[8]. Veglio M, Borra M, Stevens LK et al. The relationship between QTc interval prolongation and diabetic complications : the EURODIAB IDDM Complication Study Group. Diabetologia 1999;42:68-75.

[9]. Naohiko Takahashia et al Regulation of QT indices mediated by autonomic nervous function in patients with type 2 diabetes, International Journal of Cardiology 96(2004) 375-379.

[10]. Arduino A, Mark T, Cameron G, Stephen H. Impact of age on QT Interval and QT dispersion in healthy subjects: a regression analysis. Age and Ageing. 2003;32:326-31. 
[11]. Macfarlane PW, Lawrie TDv. The normal electrocardiogram and vectorcardiogram. In: Macfralane PW, Lawrie TDV, eds. Comprehensive Electrocardiocardiology. Oxford, UK. Pergamon Press; 1998; 98 :2160-7.

[12]. Gupta S, Epari V, Dohare S, Mane A. A study of QT interval of healthy individuals and its determinant in Indian setting.

Comparison Of Different Variables In Diabetics (N=100) And Non Diabetics (N=50) With Prolonged QTd

\begin{tabular}{|c|c|c|c|c|c|}
\hline Variables [QTD Prolonged] & Groups & No of patients & Mean & $\pm \mathrm{SD}$ & p value \\
\hline \multirow{2}{*}{ AGE } & Cases & 33 & 50.76 & 6.58 & \multirow[t]{2}{*}{0.486} \\
\hline & Controls & 6 & 48.50 & 10.50 & \\
\hline \multirow{2}{*}{ DURATION(in months) } & Cases & 33 & 40.45 & 31.72 & \multirow[t]{2}{*}{0.004} \\
\hline & Controls & 6 & 0.00 & 0.00 & \\
\hline \multirow{2}{*}{ WT(In Kgs) } & Cases & 33 & 78.25 & 9.40 & \multirow[t]{2}{*}{0.56} \\
\hline & Controls & 6 & 75.83 & 8.73 & \\
\hline \multirow{2}{*}{$\mathrm{HT}(\mathrm{cms})$} & Cases & 33 & 171.12 & 5.76 & \multirow[t]{2}{*}{0.16} \\
\hline & Controls & 6 & 167.67 & 3.93 & \\
\hline \multirow{2}{*}{ BMI } & Cases & 33 & 26.82 & 3.30 & \multirow[t]{2}{*}{0.91} \\
\hline & Controls & 6 & 26.99 & 3.40 & \\
\hline \multirow{2}{*}{ FBS (mg/dl) } & Cases & 33 & 208.36 & 72.67 & \multirow[t]{2}{*}{$<0.001$} \\
\hline & Controls & 6 & 85.50 & 8.62 & \\
\hline \multirow{2}{*}{ PPBS(mg/dl) } & Cases & 33 & 361.12 & 113.19 & \multirow[t]{2}{*}{$<0.001$} \\
\hline & Controls & 6 & 124.67 & 17.47 & \\
\hline \multirow{2}{*}{ T CHOLESTEROL } & Cases & 33 & 228.15 & 66.81 & \multirow[t]{2}{*}{0.41} \\
\hline & Controls & 6 & 253.83 & 65.56 & \\
\hline \multirow{2}{*}{ TRIGLYCERIDES } & Cases & 33 & 145.82 & 38.28 & \multirow[t]{2}{*}{0.023} \\
\hline & Controls & 6 & 187.67 & 48.88 & \\
\hline \multirow{2}{*}{ LDL } & Cases & 33 & 122.09 & 25.59 & \multirow[t]{2}{*}{0.104} \\
\hline & Controls & 6 & 141.83 & 32.85 & \\
\hline \multirow{2}{*}{ HDL } & Cases & 33 & 39.15 & 7.84 & \multirow[t]{2}{*}{0.43} \\
\hline & Controls & 6 & 36.50 & 6.02 & \\
\hline \multirow{2}{*}{ QTD(msecs) } & Cases & 33 & 0.09 & 0.01 & \multirow[t]{2}{*}{0.103} \\
\hline & Controls & 6 & 0.08 & 0.00 & \\
\hline
\end{tabular}

Comparison Of Different Variables In Diabetics (N=100) And Non Diabetics (N=50) With Normal QTd

\begin{tabular}{|c|c|c|c|c|c|}
\hline Variables [QTD Normal] & Groups & No of patients & Mean & $\pm \mathrm{SD}$ & p value \\
\hline \multirow{2}{*}{ AGE } & Cases & 67 & 46.96 & 9.99 & \multirow[t]{2}{*}{0.404} \\
\hline & Controls & 44 & 48.50 & 8.73 & \\
\hline \multirow{2}{*}{ DURATION(in months) } & Cases & 67 & 39.93 & 35.34 & \multirow[t]{2}{*}{$<0.001$} \\
\hline & Controls & 44 & 0.00 & 0.00 & \\
\hline \multirow{2}{*}{ WT(In Kgs) } & Cases & 67 & 72.60 & 10.61 & \multirow[t]{2}{*}{0.033} \\
\hline & Controls & 44 & 68.23 & 10.15 & \\
\hline \multirow{2}{*}{$\mathrm{HT}(\mathrm{cms})$} & Cases & 67 & 169.10 & 6.76 & \multirow[t]{2}{*}{0.12} \\
\hline & Controls & 44 & 167.14 & 5.91 & \\
\hline \multirow{2}{*}{ BMI } & Cases & 67 & 25.40 & 3.12 & \multirow[t]{2}{*}{0.056} \\
\hline & Controls & 44 & 24.28 & 2.71 & \\
\hline \multirow{2}{*}{ FBS (mg/dl) } & Cases & 67 & 181.61 & 52.81 & \multirow[t]{2}{*}{$<0.001$} \\
\hline & Controls & 44 & 83.23 & 11.57 & \\
\hline \multirow{2}{*}{ PPBS(mg/dl) } & Cases & 67 & 319.64 & 96.98 & \multirow[t]{2}{*}{$<0.001$} \\
\hline & Controls & 44 & 126.18 & 17.72 & \\
\hline \multirow{2}{*}{ T CHOLESTEROL } & Cases & 67 & 244.84 & 70.71 & \multirow[t]{2}{*}{0.72} \\
\hline & Controls & 44 & 239.98 & 67.93 & \\
\hline \multirow{2}{*}{ TRIGLYCERIDES } & Cases & 67 & 153.72 & 40.01 & \multirow[t]{2}{*}{0.707} \\
\hline & Controls & 44 & 150.82 & 38.87 & \\
\hline \multirow{2}{*}{ LDL } & Cases & 67 & 131.48 & 32.61 & \multirow[t]{2}{*}{0.39} \\
\hline & Controls & 44 & 126.32 & 28.11 & \\
\hline \multirow{2}{*}{ HDL } & Cases & 67 & 36.63 & 7.83 & \multirow[t]{2}{*}{0.82} \\
\hline & Controls & 44 & 36.95 & 7.74 & \\
\hline \multirow{2}{*}{ QTD(msecs) } & Cases & 67 & 0.05 & 0.02 & \multirow[t]{2}{*}{0.044} \\
\hline & Controls & 44 & 0.04 & 0.02 & \\
\hline
\end{tabular}

